\title{
Análise da Função Cognitiva e Capacidade Funcional em Idosos Hipertensos
}

\author{
Analysis of the CogritiveFuntionandPhysica FitnessinHypertensiveEdely
}

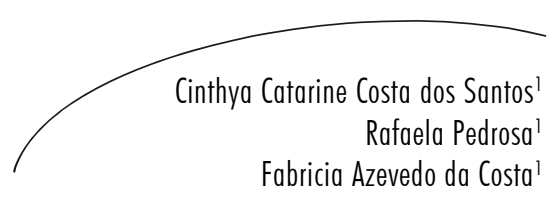

Karla Morganna Pereira Pinto de Mendonça' Gardênia Maria Holanda'

Resumo

A alteração da pressão arterial no idoso vem sendo estudada como sendo um fator de risco para o déficit na cognição e na capacidade funcional. Objetivos: investigar a função cognitiva e a capacidade funcional e sua correlação em idosos hipertensos (GH) e normotensos (GN). Métodos: estudo observacional, analítico e transversal, com 56 idosos divididos em dois grupos GH $(n=42)$ e GN $(n=14)$ avaliados através do Mini-Exame do Estado Mental (MEEM), Questionário Internacional de Atividade Física (IPAQ) e bateria de testes de aptidão física (Functional Fittness Test). Análise estatistica: os dados foram avaliados utilizando o teste $t$ de Student, o teste do Qui-quadrado e o teste de correlação de Pearson através do SPSS 17.0, sendo atribuído o nível de significância de 5\% para todos os testes. Resultados: houve correlação negativa, porém fraca entre os grupos GH e GN quanto ao déficit cognitivo $(r=-0,23 ; p=0,08)$, força de MMII $(r=-0,24 ; p=0,08)$ e a endurance aeróbica - TME2' $(r=-0,31$ e $\mathrm{p}=0,02)$. Em relação aos testes de flexibilidade de MMSS e MMII, houve correlação negativa moderada entre o grupo GH e GN $(r=-0,62 ; p<0,001$ e $r=-0,39 ; p=0,003)$ e correlação positiva moderada para agilidade e equilíbrio - TUG $(r=0,38$ e $p=0,004)$. Foi encontrada diferença significativa para o nível de atividade física entre GH e GN com $\mathrm{p}<0,001$. Conclusões: esses achados sugerem que a hipertensão arterial afeta diretamente o idoso na medida em que predispõe o declínio cognitivo e limita a capacidade do indivíduo de realizar suas atividades básicas de forma independente, diminuindo a mobilidade funcional principalmente se associada a outras comorbidades e a hábitos de vida não saudáveis.

\section{Abstract}

The change in blood pressure in the elderly has been studied as a risk factor for deficits in cognition and functional capacity. Objectives: to investigate the cognitive function and functional capacity and their correlation in elderly hypertensive (HG) and normentensive (NG). Methods: observational, analytical and crosssectional study with 56 elderly divided into two groups HG $(n=42)$ and NG
Palavras-chave: Saúde do idoso. Pressão arterial. Hábitos. Hipertensão. Envelhecimento. Cognição. Aptidão Física.

\footnotetext{
1 Curso de Fisioterapia da Universidade Federal do Rio Grande do Norte. Natal, RN, Brasil.
} 
$(\mathrm{n}=14)$ assessed by the Mini-Mental State Examination (MMSE), IPAQ (International Physical Activity Questionnaire) and Functional Fitness Test. Statistical analysis: data were evaluated using the $t$ Student test, the chi-square and Pearson's correlation test by SPSS 17.0, assigning a significance level of $5 \%$ for all tests. Results: there was a negative correlation, but low between the groups HG and NG on the cognitive deficit $(r=-0,23 ; p=0,08)$, strength of lower limbs $(r=-$ $0,24 ; p=0,08)$ and endurance aerobic - 2MST $(r=0,31 ; p=0,02)$. Testing the flexibility of upper and lower limbs there were moderately negative correlation between HG and NG groups $(r=0.62 ; p<0.001$ and $r=0.39, p=0.003)$, and positive for agility and balance - TUG $(r=0.38$ and $p=0.004)$. We found a significant difference to the level of physical activity of $H G$ and NG with $p<0.001$. Conclusions: These findings suggest that hypertension directly affects the elderly in that it predisposes to cognitive decline, and limits the ability of individuals to perform their basic activities independently, reducing the functional mobility, mainly when associated with other comorbidities and unhealthy life style.
Key words: Blood Pressure. Health of the Elderly. Habits.

Hypertension. Aging. Cognition. Physical Fitness.

\section{INTRODUÇÃO}

As alterações morfofuncionais inerentes ao processo de envelhecimento humano, quando associadas a doenças crônicas, podem levar à diminuição da independência física do idoso., ${ }^{1,2}$ A prevenção e o controle das doenças crônicas, incluindo a hipertensão arterial, podem auxiliar na manutenção da cognição ${ }^{3} \mathrm{e}$ da capacidade funcional. ${ }^{4}$

Estudos clínicos afirmam que o processo de envelhecimento, associado à presença de doenças crônicas, causa danos à função cognitiva, afetando componentes como a linguagem escrita e falada, aprendizagem, habilidades motoras, capacidade de raciocínio e de memória. ${ }^{5,6}$ No entanto, Alves \& Filho ${ }^{7}$ relatam que a pressão arterial, sendo baixa, poderia ser um agravante aos déficits cognitivos.

Para mapear comprometimento cognitivo, o Mini-Exame do Estado Mental (MEEM), traduzido e validado no Brasil por Bertolucci et al., é a escala mais utilizada tanto em pesquisas como na prática clínica, para verificar a evolução da função cognitiva e analisar a efetividade do tratamento. ${ }^{8}$

Em relação à capacidade funcional, diversos estudos demonstraram associações entre doenças crônicas e incapacidade para realização das atividades da vida diária (AVDs) nos idosos, sendo a hipertensão arterial a condição crônica mais frequente nessa faixa etária. ${ }^{4,9}$ Diante das atuais tendências de envelhecimento populacional, cresce a importância de se avaliar o estado de saúde dos idosos, identificando as doenças incapacitantes para cada indivíduo, de forma que a qualidade de vida lhe seja assegurada. ${ }^{10}$

A avaliação da capacidadefuncional através de testes que simulam os gestos realizados durante as AVDs é válido para se identificar precocemente o declínio físico, elaborar programas de exercícios individualizados, observar a evolução motora e evitar a perda de independência. ${ }^{11,12}$ Rikli \& Jones ${ }^{13}$ propuseram uma bateria de testes específica para avaliar a aptidão fisica do idoso. Os testes incluem medidas de força, flexibilidade, endurance cardiorespiratória, aptidão motora e composição corporal.

Com base no pressuposto teórico e considerando a escassez de pesquisas sobre a correlação entre função cognitiva, capacidade funcional e hipertensão, o presente estudo tem como objetivo verificar a associação entre pressão arterial sistêmica, cognição e capacidade funcional em idosos.

\section{MÉTODOS}

Estudo observacional analítico do tipo transversal foi realizado com uma amostra 
composta de 75 sujeitos, de ambos os sexos, com mais de 60 anos, alocados aleatoriamente. Os voluntários foram divididos em dois grupos, sendo o grupo GH composto por idosos hipertensos participantes do Programa de Assistência e Cuidados para o Controle da Hipertensão Arterial do Hospital Universitário Onofre Lopes (PACHA), e o grupo GN, por idosos normotensos residentes na comunidade.

Foram selecionados ao acaso 50 sujeitos do PACHA e 25 indivíduos da comunidade, que se enquadravam nos seguintes critérios: ter idade maior ou igual a 60 anos, não ser portador de pneumopatias e/ou insuficiência cardíaca, não ser consumidor de bebida alcoólica, não usar calmantes ou sedativos e não fumar há pelo menos 15 anos. Além dos critérios acima mencionados, os idosos do $\mathrm{GH}$ teriam que possuir diagnóstico clínico de hipertensão arterial há pelo menos cinco anos. Os sujeitos que estavam impossibilitados de realizar os testes funcionais e os idosos que apresentavam quadro demencial comprovado foram excluídos da pesquisa.

Todos os participantes da pesquisa assinaram o termo de consentimento livre e esclarecido e os procedimentos experimentais foram executados seguindo as normas éticas previstas na Resolução n 196, de 10/10/1996, do Conselho Nacional de Saúde. O estudo foi submetido e aprovado pelo Comitê de Ética em pesquisa da Universidade Federal do Rio Grande do Norte sob o protocolo número 209/08.

Para coleta de dados, foi realizado inicialmente o preenchimento de uma ficha de avaliação padronizada contendo perguntas sobre antecedentes patológicos e hábitos de vida. Em seguida, foram aferidos a altura e o peso, através de balança digital com estadiômetro da marca Soenhle ${ }^{\circledR}$, com capacidade para $180 \mathrm{Kg}$ e graduação de $100 \mathrm{~g}$. A pressão arterial foi aferida com o tensiômetro digital de braço Omron M6. Após a verificação do perfil do paciente, os questionários para a avaliação da função cognitiva, do nível de atividade física e da capacidade funcional foram aplicados pelo mesmo examinador, seguindo sempre a sequência abaixo definida.

\section{Avaliação Cognitiva}

O Mini-Exame do Estado Mental proposto por Foistein, Foistein \& McHugh (1975), validado no Brasil por Bertolucci et al. em 1994, foi aplicado com o objetivo de determinar o nível cognitivo dos idosos. Esse instrumento é composto por questões agrupadas em sete categorias: orientação temporal (5 pontos), orientação espacial (5 pontos), memória imediata (3 pontos), atenção e cálculo (5 pontos), evocação ( 3 pontos), linguagem (5 pontos) e praxia construcional (1 ponto). Aqueles que apresentaram pontuação do MEEM abaixo de 20 para analfabetos e abaixo de 24 para indivíduos escolarizados foram considerados como portadores de déficit cognitivo. ${ }^{15}$

\section{Avaliação do nível de atividade física}

Para avaliar duração, frequência, intensidade e tipo de atividade a que o idoso se submete durante sua rotina semanal, foi aplicado o Questionário Internacional de Atividade Física (IPAQ - versão curta), proposto pela Organização Mundial da Saúde (1998). Esse instrumento é bastante utilizado por ser de fácil preenchimento, sendo uma ótima ferramenta para diagnosticar o nível de atividade física da população e prevenir doenças causadas pela inatividade. ${ }^{14}$

\section{Avaliação da capacidade funcional}

Por último, foi testado o nível de aptidão funcional do idoso através da bateria de testes proposta por Rikli \& Jones (Functional Fitness Test). Esses testes incluem medidas de força e flexibilidade de membros superiores e inferiores, equilíbrio, endurance cardiorespiratória, aptidão motora e composição corporal. ${ }^{13}$

O Functional Fitness Testé composto dos seguintes testes:

- Sentar e levantar da cadeira em 30 segundos: o teste para verificar o nível de força dos membros inferiores é iniciado com o participante sentado no meio de uma 
cadeira com tronco ereto, pés apoiados no chão e braços cruzados sobre o peito. $\mathrm{O}$ idoso foi encorajado a levantar e sentar na cadeira o máximo de vezes possível durante 30 segundos.

- Flexão e extensão de cotovelo: esse teste avalia a força dos membros superiores. $\mathrm{O}$ participante senta na cadeira com as costas eretas, pés apoiados no chão e o lado dominante do corpo próximo à borda do assento, devendo fletir e estender o cotovelo, o máximo de vezes durante 30 segundos, segurando um halter $(2,3 \mathrm{~kg}$ para mulheres e 3,6 kg para homens).

- Marcha estacionária de dois minutos (TME2'): tem a finalidade de avaliar a endurance aeróbica.

- Deve-se quantificar o número máximo de elevações do joelho durante dois minutos. A altura do joelho apropriada para cada sujeito é nivelada em um ponto médio entre a patela e a espinha ilíaca ântero-superior.

- Teste sentar e alcançar: tem como objetivo avaliar a flexibilidade de tronco. $\mathrm{O}$ sujeito permanece sentado na cadeira e com uma perna estendida, tenta alcançar os dedos dos pés.

- Alcance das mãos atrás do tronco: avalia a flexibilidade de membros superiores. Em pé, o avaliado coloca a mão preferida sobre o mesmo ombro, a palma aberta e os dedos estendidos, alcançando o meio das costas tanto quanto possível. A mão do outro braço é colocada atrás das costas, a palma para cima, alcançando o mais distante possível, na tentativa de tocar ou sobrepor os dedos médios estendidos da outra mão.

- Levantar e caminhar ("Timed Up and Go" TUG): para avaliar a agilidade e o equilíbrio dinâmico. O indivíduo levanta da cadeira e caminha uma distância de três metros o mais rapidamente possível, sem correr e dar uma volta em torno de um cone e retorna para a cadeira.
Também compõe a bateria de testes de Rikli \& Jones o teste da caminhada dos seis minutos (TC6'). Em virtude de o TC6' e o teste da marcha estacionária (TME2') serem capazes de avaliar a endurance aeróbia do mesmo modo, ${ }^{16}$ foi escolhido utilizar neste estudo só o TME2', por ser um teste mais rápido que o TC6' e por não necessitar de um espaço físico grande.

Quanto à análise dos dados, inicialmente foi realizada uma análise descritiva, através das frequências absoluta e relativa e de média e desvio padrão, sendo os resultados apresentados em forma de tabelas. Após análise da normalidade da amostra através do teste Kolmogorov-Smirnov (K-S), foi realizado o teste $t$ de Student para amostras independentes, para avaliar as diferenças entre as médias dos MEEM, Functional Physical Test e IPAQ. Em seguida, foi aplicado o teste do Quiquadrado e verificada a razão de chances (odds ratio) para presença de declínio da função cognitiva. Foi aplicado também para comparação entre grupos de pacientes hipertensos e não hipertensos o teste de correlação de Pearson, para avaliar a associação entre a função cognitiva e a capacidade funcional. Para a análise inferencial, utilizou-se o programa SPSS 17.0 (Statistical Package for the Social Science), atribuindo o nível de significância de $5 \%$ para todos os testes estatísticos.

\section{RESULTADOS}

A amostra selecionada era inicialmente composta de 75 idosos, dos quais 50 eram hipertensos $(\mathrm{GH})$ e 25 normotensos (GN). Durante o estudo, houve perda amostral por desistência (1 idoso hipertenso e 6 normotensos) e por impossibilidade física de realizar os testes propostos (7 idosos hipertensos e 5 normotensos). Dessa forma, o $\mathrm{GH}$ apresentou $\mathrm{n}=42$, com média de idade de $65,4 \pm 5,2$, e o $G N$ apresentou $n=14$, com idade variando entre $67,4 \pm 3,9$. A amostra final do estudo foi de 56 idosos, com idade variando de 60 a 80 anos e média de 65,9 \pm 4,9 , sendo $75 \%$ da amostra compostos por hipertensos $(\mathrm{GH})$ e $25 \%$ por normotensos 
(GN). O GH era composto por $86 \%$ de mulheres e $14 \%$ de homens, enquanto o GN era por $57 \%$ de mulheres e $43 \%$ de homens. Em relação à idade e à escolaridade (medida em anos de estudo), o GN apresentou maiores médias quando comparado com o $\mathrm{GH}$.
As características sociodemográficas e relacionadas à saúde, como índice de massa corpórea (IMC), prática de atividade física em horas por dia, número de comorbidades e quantidade de medicamentos apresentaram maior média nos idosos do GH, como demonstrado na tabela 1.

Tabela 1 - Características sociodemográficas e relacionadas à saúde. Natal, RN. 2009.

\begin{tabular}{lcc}
\hline \multicolumn{2}{c}{ GH } & GN \\
\hline Idade (anos) & $65,4 \pm 5,2$ & $67,4 \pm 3,9$ \\
IMC (Kg/m ${ }^{2}$ & $28,6 \pm 5,4$ & $25,2 \pm 1,9$ \\
Escolaridade(anos) & $4,3 \pm 3,6$ & $6,7 \pm 5,1$ \\
Ativ. física (hs) & $0,8 \pm 0,7$ & $0,4 \pm 0,5$ \\
Tempo HAS(anos) & $20,3 \pm 7,9$ & 0 \\
Tempo Tto (anos) & $13,5 \pm 4,9$ & 0 \\
Comorbidades(n $\left.\mathrm{n}^{\circ}\right)$ & $1,6 \pm 1,0$ & $0,4 \pm 0,5$ \\
Medicamentos( $\left.\mathrm{n}^{\circ}\right)$ & $2,5 \pm 1,3$ & 0 \\
\hline
\end{tabular}

GH-Grupo Hipertensos ( $\mathrm{n}=42)$; GN-Grupo Normotensos $(\mathrm{n}=14)$.

Com a avaliação da função cognitiva da amostra através do MEEM, foi verificado que $69 \%$ dos hipertensos apresentaram declínio cognitivo, ou seja, 29 idosos do $\mathrm{GH}$ não atingiram a pontuação mínima de 20 pontos para indivíduos analfabetos e de 24 para escolarizados. Comparando a resposta obtida no GN com a resposta do $\mathrm{GH}$, observou-se uma diferença marginalmente significante $(\mathrm{p}=0,08)$, uma vez que apenas $43 \%$ dos indivíduos normotensos apresentaram déficit cognitivo. Além disso, observamos que as médias de pontuação no MEEM para o GN e o GH foram bem parecidas $(23,2 \pm 3,0$ e $22,7 \pm 2,3$, respectivamente), não havendo diferença estatística entre elas.

A associação da HAS com a pontuação global do MEEM não apresentou correlação $(r=-0,047$ e $\mathrm{p}=0,73$ ), mas houve correlação negativa de fraca a moderada entre a pressão arterial diastólica (PAD) e a pontuação final do MEEM verificado por $(r=-0,289$ e $\mathrm{p}=0,031)$. Analisando a resposta dos idosos para cada domínio do MEEM, não foi encontrada diferença significativa entre atenção e cálculo $(p=0,08)$, a memória $(p=0,43)$ e a praxia construcional $(p=0,53)$.

Em relação ao nível de atividade física, medida através do IPAQ, os idosos do GH tiveram médias de respostas melhores que os idosos do GN em quase todos os quesitos, com exceção dos relacionados a atividades moderadas, onde houve diferença marginalmente significativa $(\mathrm{p}=0,08)$ entre as respostas dos sujeitos do $\mathrm{GH}(3,4 \pm 2,8$ dias), para o subitem horas/dia, quando comparado com os do GN. No entanto, em relação ao subitem quantidade de dias dedicados à realização de atividades moderadas na última semana, não houve diferença significativa. Como mostra a tabela 2, o tempo em que os idosos permanecem sentados durante a semana foi maior para os sujeitos do grupo GN, sendo a diferença significativa entre os grupos, evidenciando que os normotensos permanecem mais tempo fazendo atividades que não exigem grandes gastos de energia e não trazem benefícios significativos aos sistemas cardiovascular, respiratório e músculoesquelético. 
Tabela 2 - Distribuição das médias do IPAQ*. Natal, RN. 2009.

\begin{tabular}{lccc}
\hline & GH & GN & p \\
\hline Caminhada (dias) & $3,4 \pm 2,6$ & $2,4 \pm 2,7$ & 0,221 \\
Caminhada (hs/dia) & $1 \pm 2,33$ & $0,4 \pm 0,4$ & 0,352 \\
Atividades moderadas (dias) & $5,2 \pm 2,4$ & $5,5 \pm 2,1$ & 0,666 \\
Atividades moderadas (hs/dia) & $3,4 \pm 2,8$ & $4,9 \pm 2,8$ & 0,082 \\
Atividades vigor (dias) & $0,9 \pm 2,0$ & $0,2 \pm 0,6$ & 0,194 \\
Atividades vigor (hs/dia) & $0,3 \pm 0,7$ & $0,3 \pm 0,7$ & 0,663 \\
Sentado (semana) & $4,4 \pm 1,9$ & $6,3 \pm 5,6$ & 0,025 \\
Sentado (sáb/dom) & $4,1 \pm 1,8$ & $4,6 \pm 1,9$ & 0,433 \\
\hline
\end{tabular}

*IPAQ (Internacional Physical Activity Questionnaire).

GH-Grupo Hipertensos ( $\mathrm{n}=42)$; GN-Grupo Normotensos $(\mathrm{n}=14)$

Ao se analisarem os resultados dos testes de aptidão física, os normotensos obtiveram melhores médias em todos os itens, sobretudo nos testes de mobilidade e flexibilidade, onde as diferenças entre os grupos $\mathrm{GH}$ e $\mathrm{GN}$ foram significativas para o TUG e o TME2' e para o teste de flexibilidade em MMII (teste sentaralcançar) e MMSS (teste de alcance das mãos). Em relação aos testes de força para MMSS (Flexão de cotovelo) e de MMII (sentar-levantar), não houve diferença significativa para as respostas dos dois grupos, como mostra a tabela 3.

Tabela 3 - Distribuição das médias dos testes de Aptidão Física*. Natal, RN. 2009.

\begin{tabular}{lccc}
\hline & GH & GN & p \\
\hline Sentar - levantar (ciclos) & $9,3 \pm 2,1$ & $10,6 \pm 2,6$ & 0,078 \\
Fletir cotovelo (flexões) & $10,2 \pm 3,0$ & $11,2 \pm 2,7$ & 0,277 \\
Sentar - alcançar (cm) & $-5,8 \pm 11,1$ & $-3,5 \pm 11,2$ & 0,003 \\
Alcance das mãos (cm) & $-7,5 \pm 13,3$ & $-1,6 \pm 9,7$ & $0,000 * *$ \\
TME2' (passos) & $46,7 \pm 11,8$ & $56,2 \pm 15,5$ & 0,020 \\
TUG (s) & $8,8 \pm 1,8$ & $7,3 \pm 1,5$ & 0,004
\end{tabular}

"Rikli RE,Jones CJ.(Development and validation of a functional fitness test for community-residing older adults, 1999). GH-Grupo Hipertensos $(\mathrm{n}=42)$; GN-Grupo Normotensos $(\mathrm{n}=14)$. ** $\mathrm{p}<0,001$

Com a correlação entre os testes de flexibilidade e a HAS, foi observada associação negativa moderada para o teste de MMII de sentaralcançar; já para MMSS, houve correlação negativa de moderada a forte, evidenciando que os hipertensos apresentaram maior dificuldade de alcançar as mãos atrás do tronco, como mostra a tabela 4. 
Tabela 4 - Correlação de Pearson entre HAS e Functional Pbysical Test . Natal, RN. 2009.

\begin{tabular}{lcc}
\hline & $\mathrm{r}$ & $\mathrm{p}$ \\
\hline HAS e TUG* & 0,375 & 0,004 \\
HAS e TME2* & $-0,311$ & 0,020 \\
HAS e sentar - levantar* & $-0,238$ & 0,078 \\
HAS e flexão cotovelo* & $-0,148$ & 0,277 \\
HAS e sentar - alcançar* & 0,389 & 0,003 \\
HAS e alcance das mãos* & 0,619 & 0,000 \\
\hline
\end{tabular}

"Functional Physical Test (Rikli e Jones, 1999); TUG: Teste Time Up and Go; TME2':

Teste da Marcha Estacionária dos 2 min. $(\mathrm{n}=56)$.

\section{DISCUSSÃO}

Diante do crescente aumento na expectativa de vida da população mundial e a prevalência de doenças associadas ao processo de senescência, é de suma importância avaliar o idoso sob o ponto de vista cognitivo e funcional, com o objetivo de prevenir e/ou minimizar os efeitos degenerativos inerentes ao envelhecimento, propiciando melhora na qualidade de vida do idoso. ${ }^{17}$

Existem vários instrumentos capazes de avaliar a função cognitiva no idoso, sendo o Mini-Exame do Estado Mental a escala mais utilizada para mapear o déficit cognitivo, por ser de fácil aplicação, baixo custo e possuir sensibilidade e especificidade adequada. No presente estudo, foi aplicado o MEEM em 56 idosos divididos em dois grupos não pareados (normotensos - $\mathrm{GN} \mathrm{e}$ hipertensos - GH).

De acordo com as respostas obtidas no teste, observou-se declínio cognitivo maior e mais frequente nos hipertensos, corroborando outros estudos transversais e longitudinais, que verificaram baixo desempenho cognitivo em indivíduos hipertensos ou com pressão arterial sistólica isolada (PASi) aumentada. ${ }^{18,19}$

$\mathrm{Na}$ associação entre a HAS e a pontuação global do MEEM, não houve correlação, mas houve associação entre a pressão arterial diastólica (PAD) e a pontuação final do MEEM. Esse fato também foi observado por Reinprencht et al., ${ }^{20}$ ao acompanharem 186 idosos durante um estudo de seguimento de 13 anos. Os autores encontraram uma relação inversa entre a PAD e o escore do MEEM, principalmente nos domínios que envolvem orientação temporal, espacial e memória. Guo et al. ${ }^{21}$ observaram que a hipertensão arterial não controlada é um risco para a função cognitiva, podendo aumentar ainda mais o déficit cognitivo quando associada a diabetes mellitus, tabagismo, álcool e obesidade. ${ }^{22}$ Isso corrobora diversos estudos que apontam para o fato de a hipertensão acelerar o processo de perda cognitiva, devido ao efeito deletério sobre a substância branca cerebral e na função cerebrovascular. ${ }^{5,623}$

Em relação à resposta dos idosos para cada domínio do MEEM, não foi observada diferença significativa entre atenção e cálculo e os domínios memória e construção visual. Esse fato se deve, provavelmente, à influência da educação ${ }^{24} \mathrm{e}$ da faixa etária dos sujeitos. ${ }^{25}$ Para diminuir o efeito adverso do baixo nível de escolaridade, foram propostos pontos de corte diferentes para os indivíduos não-escolarizados e escolarizados, de forma que o instrumento detecte apenas os domínios que não obtiveram resposta adequada devido ao declínio cognitivo. ${ }^{17}$

Em virtude de os sujeitos do grupo GH serem participantes de um programa de cuidados com a hipertensão arterial, no qual são estimulados a praticar atividade física, orientados a seguir dieta hipossódica, hipolipídica e hipocalórica, não se 
verificou diferença significativa entre o grupos em relação aos testes de força para membros superiores (teste de flexão de cotovelo) e inferiores (teste senta-levanta), evidenciando os efeitos benéficos do exercício na manutenção da força e do trofismo muscular nos indivíduos hipertensos fisicamente ativos.

No entanto, em relação ao TME2', houve diferença significativa. Isso pode ser explicado pelo fato de desse teste ser um tipo de atividade vigorosa, que promove aumento do gasto energético, que previamente já era alto, aumento da pressão arterial, sobretudo a sistólica, aumento da frequência respiratória e cardíaca. Além disso, apesar de se tratar de idosos ativos, no grupo dos hipertensos existe uma diminuição da aptidão cardiorrespiratória, causada pela associação da HAS a comorbidades como diabetes mellitus e insuficiência cardíaca. ${ }^{26}$

O TUG também apresentou diferença significante entre os dois grupos de idosos, porque esse teste requer agilidade e equilíbrio, características que estavam mais afetadas no $\mathrm{GH}$ devido à maior frequência de comorbidades nesse grupo. $O$ fato de os hipertensos praticarem mais atividade física que os idosos do GN não diminuiu o tempo necessário para completar o teste, pois eles realizam atividades predominantemente aeróbicas, como caminhada e hidroginástica, porém negligenciam os exercícios que propiciam melhora na potência muscular e no equilíbrio. ${ }^{27}$

Em relação aos testes de flexibilidade, houve correlação moderada para o teste sentar-alcançar, demonstrando que os hipertensos apresentam menor flexibilidade em MMII, sobretudo nos músculos da panturilha. Para flexibilidade em MMSS, existiu associação de moderada a forte ou seja, os hipertensos apresentaram maior dificuldade de alcançar as mãos atrás do tronco, devido à diminuição da flexibilidade dos músculos da articulação glenoumeral. Essa diferença provavelmente seja causada por comorbidades, em especial as doenças reumatológicas, que foram mais prevalentes nos idosos do grupo GH.

Por fim, foi verificado que os idosos hipertensos possuem maior risco de apresentarem declínio da função cognitiva, quando comparados aos idosos do GN. Esse risco também foi evidenciado por Naranjo \& Moreno, ${ }^{28}$ os quais encontraram uma razão de chance de $4 \%$ para os idosos hipertensos em relação aos idosos saudáveis. ${ }^{21}$ No entanto, Guo et al. ${ }^{21}$ verificaram que indivíduos com pressão arterial sistólica menor que $130 \mathrm{mmHg}$ apresentaram risco de $1,88 \%$.

Este estudo apresentou como limitação o pequeno número amostral do grupo considerado controle, de idosos normotensos, podendo ter comprometido, em especial, o rastreamento do declínio cognitivo. Sugere-se que novos estudos sejam realizados, com maior número amostral, avaliando a influência e os efeitos deletérios da hipertensão arterial na função cognitiva do sujeito idoso, a fim de tornar a literatura consensual.

\section{CONCLUSÃO}

Diante dos resultados encontrados neste estudo e na literatura, sugerimos que a hipertensão afeta diretamente o idoso na medida em que limita a capacidade do indivíduo de realizar suas atividades básicas de forma independente, diminuindo a mobilidade funcional, sobretudo se associada a outras comorbidades e hábitos de vida não saudáveis. Além disso, o estudo indica que a hipertensão arterial apresenta um efeito deletério na cognição.

Assim, para que o idoso continue inserido na sociedade de forma ativa, é necessário que existam mais programas de prevenção e combate das doenças crônicas degenerativas, principalmente a hipertensão arterial, de forma que o idoso mantenha a cognição preservada e consiga realizar as AVDs de forma independente. 


\section{REFERÊNCIAS}

1. Matsudo S, Araújo T, Matsudo V, Andrade D, Andrade E, Oliveira LC, Braggion G. Questionário internacional de atividade física : estudo de validade e reprodutibilidade no brasil. Revi Bras Ativ Fís Saúde 2001;6 (2):05-12.

2. Rosa TEC, Benício MHA, Latorre MRDO, Ramos LR. Fatores determinantes da capacidade funcional entre idosos. Rev Saúd Pública 2003; 37(1):408.

3. Knecht S, Wersching H, Lohmann H, Berger K, Ringelstein EB. How much does hypertension affect cognition?. J Neurol Sci 2009;283(1-2):149-52.

4. Alves LC, Leimann BCQ, Vasconcelos MEL, Carvalho MS, Vasconcelos AGG, TCO Fonseca, Lebrão ML, Laurenti R. A influência das doenças crônicas na capacidade funcional dos idosos do Município de São Paulo, Brasil. Cad. Saúd Pública 2007;23(8):1924-30.

5. Birns J, Kalra L. Cognitive function and hypertension. J Human Hypertension 2008; 23(2):86-96.

6. Melo ROV, Martin JFV.Influência dos níveis pressóricos no desenvolvimento do déficit cognitivo. Rev Bras Hipertens 2008;15(1):37-8.

7. Alves TCTF, Filho GB, Wajngarten M. Transtornos Cognitivos e Hipertensão. Rev Soc Bras Hipertensão 2003;6(1):48.

8. Almeida OP. Mini Exame do estado mental e o diagnóstico de demência no Brasil. Arq Neuropsiquiatr 1998;56(3-b):605-12.

9. Hajjar I, Lackland DT, Cupples LA, Lipsitz LA. Association between concurrent and remote blood pressure and disability in older adults. Hypertension 2007;50(6):1026-32.

10. Costa AJL. Metodologias e indicadores para avaliação da capacidade funcional: análise preliminar do Suplemento Saúde da Pesquisa Nacional por Amostra de Domicílios (PNAD). Ciênc Saúd Coletiva 2006;11(4)927-40.

11. Wind H, Gouttebarge V, Kuijer PPFM, Sluiter JK, Frings-Dresen MHW. The utility of functional capacity evaluation: the opinion of physicians and other experts in the field of return to work and disability claims. Int Arch Occup Environ Health 2006;79(6):528-34.

12. Maciel ACC, Guerra RO. Influência dos fatores biopsicossociais sobre a capacidade funcional de idosos residentes no nordeste do Brasil. Rev Bras Epidemiol 2007;10(2):178-89.

13. Rikli RE, Jones CJ. Developmental and validation of a functional fitness test for community-residing older adults. J Aging Phys Act 1999;7:129-61.

14. Castro KCM, Guerra RO. Impact of cognitive performance on the functional capacity of an elderly population in Natal. Arq Neuropsiquiatr 2008; 66(4):809-13.

15. Parahyba MI, Veras R, Melzer D. Incapacidade funcional entre as mulheres idosas no Brasil. Rev Saúde Pública 2005;39(3):383-91.

16. Pedrosa R, Holanda G. Correlação entre os testes da caminhada, marcha estacionária e TUG em hipertensas idosas. Rev Bras Fisioter 2009;3:252-6.

17. Amado TCF, Arruda IKG. Hipertensão arterial no idoso e fatores de risco associados. Rev Bras Nutr Clin 2004;19(2):94-9.

18. Obisesan TO, Obisesan AO, Martins S, Alamgir L, Bond V, Maxwell C, et al. High Blood Pressure, hypertension, and high pulse pressure are associated with poorer cognitive function in persons aged 60 and older : the third national health and nutrition examination survey. J Am Geriatr Soc 2008;56(3)501-9.

19. Johnson $\mathrm{KC}$, Margolis KL, Espeland MA, Colenda CC, Fillit H, Manson JE, Masaki KH ,et al. A Prospective study of the effect of hypertension and baseline blood pressure on cognitive decline and dementia in postmenopausal women: the women's health initiative memory study. J Am Geriatr Soc 2008;56(8)1449-58.

20. Reinprencht F, Elmstahl S, Janzon L, AndréPeterson L. Hypetension and changes of cognitive function in 81-year-old-men: a 13 year follow up of the population study.Men Born in 1914, Sweden. Hypertens 2003;21:57-66.

21. Guo Z, Fratiglioni L, Winblad B, Vitalen M. Blood pressure and performance on the mini mental state examination in the very old. $A m \mathrm{~J}$ Epidemiol 1997;14(5) : 1106-13.

22. Van den Berg E, Kloppenborg RP, Kessels RPC, Kappelle LJ, Biessels GJ. Type 2 diabetes mellitus, hypertension, dyslipidemia and obesity: a systematic comparison of their impact on cognition. Biochim Biophys Acta 2009;1792:470-81.

23. Surh JA, Stewart JC, France CR. The Relationship between blood pressure and cognitive performance in the third national health and nutrition examination survey (NHANES III). Psychos Med 2004;66:291-97. 
24. Brito-Marques PR, Cabral-Filho JE. Influence of age and schooling on the performance in a modified mini-mental state examination version: a study in Brazil northeast. Arq Neuropsiquiatr 2005;63(3-A):583-7.

25. Valle EA, Castro-Costa E, Firmo JOA, Uchoa E, Lima-Costa MF.Estudo de base populacional dos fatores associados ao desempenho no Mini Exame do Estado Mental entre idosos: projeto Bambuí. Cad Saúde Pública 2009;25(4):918-26.

26. Morey MC, Sloane R, Pieper CF, Peterson MJ, Pearson MP, Ekelund CC, et al. Effect of physical activity guidelines on physical function in older Adults. J Am Geriatr Soc 2008;56(10):1873-78

27. Faria JC, Machala CC, Dias RC, Dias JMD. Importância do treinamento de força na reabilitação da função muscular, equilíbrio e mobilidade de idosos. Acta Fisiátrica 2003;10(3):133-7.

28. Naranjo IC, Moreno JMR. Hipertensión arterial y función cognitiva. Med Clin 2008;130(14):542-52. 